\title{
The shortened Örebro Musculoskeletal Screening Questionnaire: evaluation in a work-injured population
}

Charles Philip Gabel ${ }^{1,2}$, MSc, PT, cp.gabel@ bigpond.com

Brendan Burkett ${ }^{1}, \mathrm{PhD}$, bburkett@usc.edu.au

Markus Melloh ${ }^{3}$, MD MPH PhD, markus.melloh@uwa.edu.au

${ }^{1}$ Faculty of Science, Health, Education and Engineering Centre for Healthy Activities, Sport and Exercise, University of the Sunshine Coast, Queensland, Australia;

${ }^{2}$ Primary Health Care Section, School of Medicine, Griffith University, Queensland, Australia;

${ }^{3}$ Western Australian Institute for Medical Research (WAIMR), University of Western Australia, Nedlands, Western Australia, Australia

Address correspondence and reprint requests to:

Charles Philip Gabel,

PO Box 760, Coolum Beach, Queensland,

Australia 4573. Email: cp.gabel@ bigpond.com

Word count: Main Text $=3285$, Abstract $=250$ 


\section{ABSTRACT}

The Örebro Musculoskeletal Screening Questionnaire (ÖMSQ) is a recently validated, 21-item instrument. It modified the original Örebro Musculoskeletal Pain Questionnaire (ÖMPainQ) providing broader focus and also improved development and practicality for identfying work-injured patients at-risk of persistent musculoskeletal problems. These instruments are critiqued for practicality and a shortenedversion recommended. A 10-item ÖMPainQ was previously proposed for low-back-pain; however, general musculoskeletal populations require a broader validated instrument. To provide this, a two-stage retrospective study was performed. Stage 1 used three phases to: determined a minimum 12-item tool was required to ensure internal consistency ( $\alpha>0.70)$; subsequently developed two shortened ÖMSQ-12 versions from qualitative content-retention and quantitative factor analysis reductive methodologies; then calibrated both versions in a spine-cohort. Stage 2 validated and compared both versions' clinimetric properties in a general musculoskeletal-cohort to ascertain which was most appropriate. The ÖMPainQ10 and a randomly-created ÖMPainQ-10 were compared post-hoc for criterion validity and factor structure. A physical therapy outpatients convenience sample $(n=279)$ was divided into developmental (spine=136) and calibration (musculoskeletal=143) cohorts. Primary outcomes were functional status, insurer-reported absenteeism and costs at six months. The qualitative-ÖMSQ-12 demonstrated preferred properties with higher 21-item-ÖMSQ correlation ( $\mathrm{r}=0.97$; quantitative-ÖMSQ-12:r=0.94; ÖMPainQ10:r=0.92; ÖMPainQ-10-random:r=0.94) and improved predictive ability cut-offs for high-risk (72 ÖMSQ-12 points, 60\%) and low-risk (57 ÖMSQ-12 points, 48\% ). The ÖMSQ-12 content-retention version is recommended. It demonstrated suitable internal consistency, a three-factor structure and high correlation with recovery time ( $\mathrm{r}=0.73)$. The ÖMSQ-12 will facilitate early identification and management of at-risk individuals and enable targeted intervention strategies through psychosocial informed management principles.

KEYWORDS: Prognosis, screening, outcome measure, musculoskeletal, occupational, injury 


\section{INTRODUCTION}

Biopsychosocial screening instruments have been used successfully in primary care over the past two decades (Melloh et al., 2009). These instruments assist clinicians with the early identification of patients with 'yellow flags' (Liebenson and Yeomans, 2007) and the potential of an increased risk of delayed recovery from musculoskeletal injury (Boersma and Linton 2005; Westman et al., 2008; Gabel et al., 2012) - including low back pain (LBP) (Hill et al., 2009; Gabel et al., 2011). This screening process is essential as the majority of financial (Driessen et al., 2008) and social (Ijzelenberg and Burdorf, 2005) costs are attributed to the small percentage of acute injuries that transition to chronicity (Gjesdal et al., 2009; Ramond et al., 2011).

An instrument developed for this screening purpose was the 'Örebro Musculoskeletal Pain Questionnaire' (ÖMPainQ) (Linton and Boersma, 2003). The ÖMPainQ is advocated within several treatment guidelines (van Tulder et al., 2006; MAA-NSW, 2012) and also supported by two systematic reviews (Hockings et al., 2008; Sattelmayer et al., 2011). However, both reviews recommended further research to improve the instrument's practicality and confirm its predictive ability. In order to fulfill these recommendations the ÖMPain Q was modified to the ‘Örebro Musculoskeletal Screening Questionnaire' (ÖMSQ) through changes to its wording and item content. These changes improved the tool's psychometric and practical characteristics as well as its predictive ability at six months. This was initially achieved through calibration in a LBP population (Gabel et al., 2011), subsequent validation in an independent whiplash population (Gabel et al., 2008) and further validation in a 'general' musculoskeletal population that included conditions affecting the spine and limbs (Gabel et al., 2012).

In each of these three studies, the results indicated the 21-item ÖMSQ had the potential to be shortened. A shorter version would improve practicality and reduce the burden on patients, clinicians and researchers. This proposition was demonstrated by the recent publication of the ÖMPainQ-10 for LBP populations (Linton et al., 2011). However, this 10-item version contained items from only four of the established six constructs (Westman et al., 2008; Gabel et al., 2011; Gabel et al., 2012) and retained wording that focused on the low back region, pain and work. It did not reflect the specific six changes recommended for the original 21-item ÖMPain $\mathrm{Q}$ (Gabel et al., 2011) that would improve its practicality, broaden its application to the general musculoskeletal population and include non-working individuals. Consequently, a shortened 
version that did account for these changes would be an advantage, particularly if it used items from all six constructs (Gabel et al., 2011; Gabel et al., 2012).

This study's primary objective was to improve the practicality of the recently published 21-item ÖMSQ by developing and validating a shortened version. This process would require determination of the minimum number of questions necessary to retain optimal internal consistency and subsequent correlation with the full original 21-item ÖMSQ. The validation process would ensure the psychometric characteristics were retained, the six constructs remained, the four themes of the World Health Organization International Classification of Functioning, Disability and Health (WHO-ICF) (World Health Organisation, 2001) were represented, and that predictive ability at six months was demonstrated.

\section{METHOD}

\section{$\underline{\text { Design }}$}

A two-stage retrospective study was conducted where Ethics approval was obtained from the University of the Sunshine Coast (Figure 1). This was a preliminary study to indicate the potential effectiveness of a short-form ÖMSQ in predicting delayed recovery. By extracting predetermined items from existing original 21-item ÖMSQ responses, a secondary analysis of the outcomes could be performed and 12 clinimetric properties, eight psychometric and four practical, could be assessed. These findings would determine if the shortened version retained the required clinimetric properties and if a future prospective investigation was warranted.

\section{Stage 1}

Stage 1 was completed in three phases. Phase-1 used the Spearman-Brown prediction formula for a-priori determination that a minimum 12-item tool was required to ensure internal consistency was retained at an acceptable level $\alpha>0.70$ for the full document (George and Mallery, 2003; Field, 2005) and $\alpha>0.65$ for the individual identified factors (Beaton, 2005).

Phase-2 used two separate reductive methodologies to develop and produce two ÖMSQ12 versions through: A) a qualitative content-retention approach; and B) a quantitative exploratory factor analysis (EFA) approach (Table 1). The qualitative concept-retention approach (Beaton et al., 2005) used a peer panel of two physiotherapists, an occupational therapist and an occupational physician; and a patient focus group of four pairs of individuals with injuries that were respectively work and non-work related that affected their neck, back, upper limb and lower limb. 
Table 1: Items and constructs of ÖMSQ-12 concept-retention and factor analysis versions, ÖMPainQ-10 and ÖMPainQ-10 from random items

\begin{tabular}{|c|c|c|c|c|c|}
\hline Question item & Construct & $\begin{array}{l}\text { Concept- } \\
\text { retention }\end{array}$ & $\begin{array}{c}\text { Factor } \\
\text { analysis }\end{array}$ & $\begin{array}{c}\text { ÖMPainQ-10 } \\
\text { Linton } 2011\end{array}$ & $\begin{array}{c}\text { ÖMPainQ -10 } \\
\text { random }\end{array}$ \\
\hline Q17 Light work - 1 hour & 1 Physical & & & Y & $\mathrm{Y}$ \\
\hline Q18 Walk or light recreational activity & 1 Physical & Y & Y & & Y \\
\hline Q19 Home activity & 1 Physical & & & Y & \\
\hline Q20 ADL \& social & 1 Physical & Y & Y & & \\
\hline Q21 Sleep or movement in bed & 1 Physical & & & & Y \\
\hline Q1 Region & 2 Problem & & & & \\
\hline Q3 Problem duration & 2 Problem & Y & Y & Y & \\
\hline Q5 Problem intensity - acute & 2 Problem & & Y & Y & \\
\hline Q6 Problem severity - chronic & 2 Problem & Y & $\mathrm{Y}$ & & $\mathrm{Y}$ \\
\hline Q7 Problem frequency & 2 Problem & $\mathrm{Y}$ & Y & & Y \\
\hline Q8 Cope with problem & 3 Psyche & & & & $\mathrm{Y}$ \\
\hline Q9 Anxiety & 3 Psyche & Y & Y & Y & $\mathrm{Y}$ \\
\hline Q10 Depression & 3 Psyche & $\mathrm{Y}$ & $\mathrm{Y}$ & $\mathrm{Y}$ & $\mathrm{Y}$ \\
\hline Q11 Recovery expectation: of problem & 3 Psyche & $\mathrm{Y}$ & & Y & $\mathrm{Y}$ \\
\hline Q12 Recovery expectation: of work & 3 Psyche & & & Y & \\
\hline Q14 Fear-avoidance: activity makes worse & 4 Fear-avoidance & Y & & & \\
\hline Q15 Fear-avoidance: stop if activity if worse & 4 Fear-avoidance & & $\mathrm{Y}$ & $\mathrm{Y}$ & \\
\hline Q16 Fear-avoidance: stop work/ ADL if worse & 4 Fear-avoidance & $\mathrm{Y}$ & $\mathrm{Y}$ & $\mathrm{Y}$ & Y \\
\hline Q4 Burdensome & 5 Other & $\mathrm{Y}$ & Y & & \\
\hline Q13 Job satisfaction & 5 Other & $\mathrm{Y}$ & & & \\
\hline Q2 Absenteeism & 6 Personal & & Y & & \\
\hline
\end{tabular}

The quantitative EFA approach used maximum likelihood extraction (MLE) (Costello and Osborne, 2005), a statistical based approach, to choose the highest loading 12 items from the original 21-item ÖMSQ in a musculoskeletal sample $(n=279)$.

Phase-3 calibrated the clinimetric properties of both ÖMSQ-12 versions from the extracted item responses in a symptomatic spine cohort $(n=136)$. This cohort combined patients from two previous studies on whiplash (n=30) (Gabel et al., 2008) and LBP (n=106) (Gabel et al., 2011). The clinimetric properties of psychometric and practical characteristics, apart from 
scoring and completion times, were determined and compared with the original 21 -item ÖMSQ as an initial indicator of which version may be preferred.

Figure 1: Flow chart for retrospective development and validation of the ÖMSQ-12

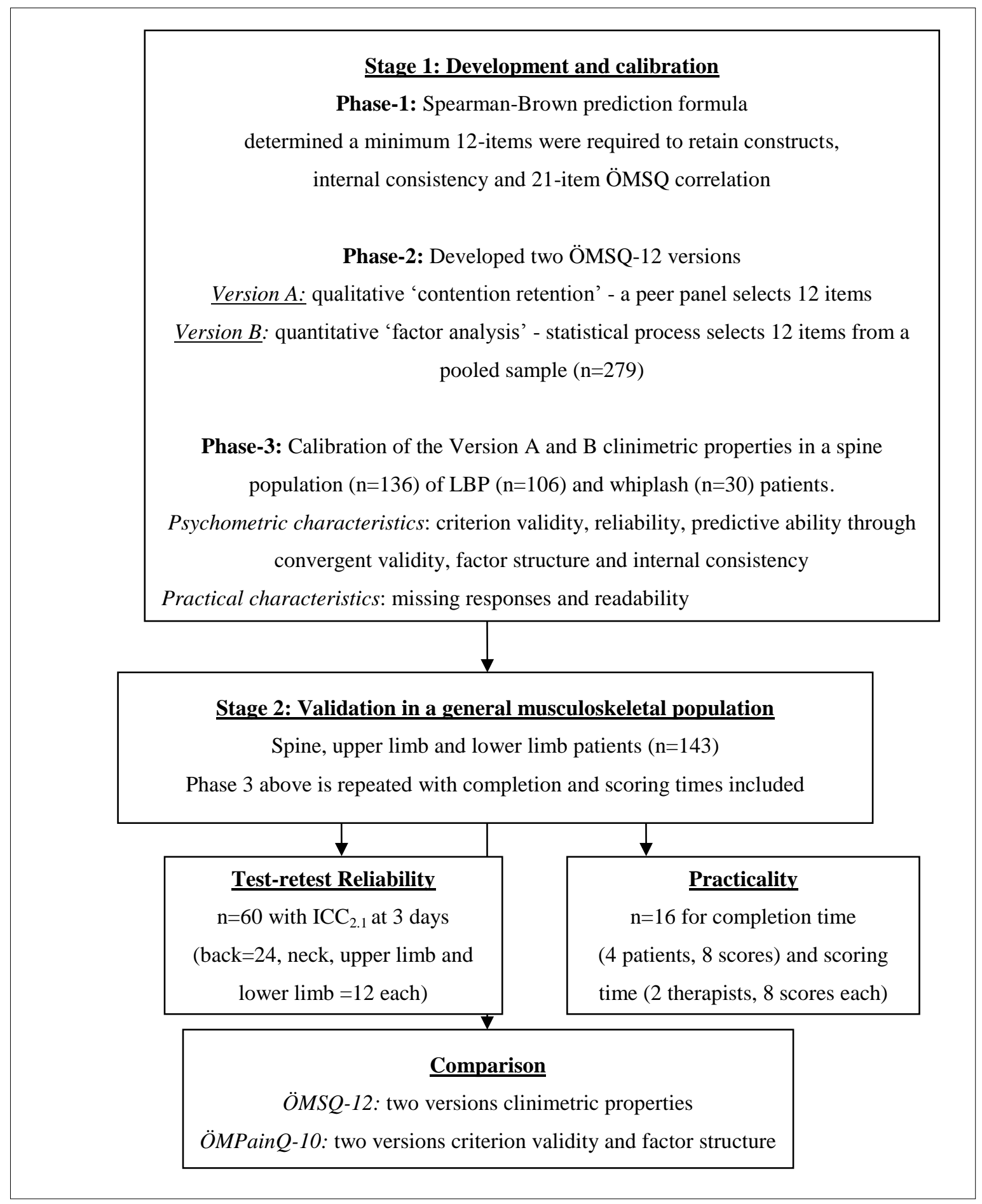

n denotes number 
A12-item Örebro Screening Questionnaire

Stage 2

Stage 2 repeated the retrospective validation process for both ÖMSQ-12 versions in a separate general musculoskeletal cohort $(n=143)$ using data extracted from the original 21-item ÖMSQ responses (Gabel et al., 2012). This sample was representative of the target population this final short-form instrument was intended - general musculoskeletal patients including those with LBP. The clinimetric properties of both ÖMSQ-12 versions were compared to each other and those previously determined for the original 21-item ÖMSQ (Gabel et al., 2012).

\section{Participants}

A total of 279 participants were involved. The 'spine' musculoskeletal data set $(n=136)$ used to develop and calibrate the ÖMSQ-12 and the 'general' musculoskeletal data set ( $\mathrm{n}=143$ ) (Gabel et al., 2012) used to validate the clinimetric properties and predictive ability at six months. Participants had work-related musculoskeletal injuries, were referred by a general practitioner to physiotherapy (Table 2) and were recruited consecutively till the minimum a-priori sample size for that respective study was achieved. Inclusion criteria were an acute/subacute musculoskeletal injury to the spine, upper limb and/or lower limb. Exclusion criteria were pregnancy, red flag features of serious pathology, age below 18 years and difficulty with English comprehension. The insurer outcome data was provided independently and the outcome assessors had been blinded to the baseline original 21-item ÖMSQ scores.

Table 2: Characteristics of the musculoskeletal calibration and validation cohorts

\begin{tabular}{|c|c|c|}
\hline Sample characteristics & ÖMSQ-12 calibration cohort & ÖMSQ-12 validation cohort \\
\hline Body region & Spine & Spine, upper and lower limbs \\
\hline $\mathbf{n}$ & 136 & 143 \\
\hline Diagnoses & Spine with/without limb referral & General \\
\hline Age (years; mean \pm SD) & $37.8 \pm 10.2$ & $39.3 \pm 9.7$ \\
\hline Gender (\% female) & 50 & 43 \\
\hline
\end{tabular}

\section{$\underline{\text { Measures and procedures }}$}

Full methodology of the original studies are detailed elsewhere (Gabel et al., 2008; Gabel et al., 2011; Gabel et al., 2012). The determination of predictive ability and the relevant clinimetric 
properties are detailed below. Baseline and follow-up regional patient reported outcome (PRO) measures for function and an 11-point numerical rating scale (NRS) for perceived problem or pain severity were taken from the original studies.

In addition, a post-hoc criterion validity and factor structure analysis was conducted and included for the recently proposed ÖMPainQ-10 (Linton et al., 2011). To ensure continuity of the comparison, a randomly generated set of ten original ÖMPainQ-items were also formed to provide a comparative control (Beaton et al., 2005) (Table 2). The ÖMPainQ-10 was published after this study was completed and analysis has been included to provide comparative details on the two psychometric properties of criterion validity and factor structure that were most influential in deciding which version of the ÖMSQ-12 was preferred.

\section{Predictive ability}

The outcomes were classified dichotomously for the presence or absence of each outcome trait:

1) not recovered at $>10 \%$;

2) long term absenteeism at $>28$ paid days off (PDO);

3) no absenteeism at $\mathrm{PDO}=0$;

4) low claim cost at $<\$ 1,000$;

5) high claim cost at $>\$ 10,000$;

6) recovery time in days to $>80 \%\left(\mathrm{t}^{80}\right)$ on the PROs.

These outcomes were cross-referenced with extracted ÖMSQ-12 scores to determine predictive ability through positive likelihood ratios.

\section{$\underline{\text { Psychometric characteristics }}$}

The eight psychometric characteristics (Gabel et al., 2011; Gabel et al., 2012) included the four aspects of validity, face and content (through the reductive process), criterion (between both ÖMSQ-12 versions and the original 21-item ÖMSQ), and construct through convergent validity (as determined from the predictive ability) and divergent validity ( $\mathrm{t}$-tests comparing screening scores between groups with known positive and negative traits for each outcome); reliability (test-retest using the $\mathrm{ICC}_{2.1}$ at three days, $\mathrm{n}=60$ with back=24, neck, upper limb and lower limb $=12$ each); recovery (correlation between baseline screening scores and the time, $\mathrm{t}^{80}$, taken to reach $80 \%$ of maximum on a regional patient reported outcome); exploratory factor analysis (through MLE) and internal consistency (through Cronbach's alpha). The original 21-item 
ÖMSQ was previously demonstrated as a six-factor questionnaire (Gabel et al., 2011; Gabel et al., 2012) with an internal consistency range of $\alpha=0.64$ to 0.81 for the individual constructs. Though a single measure of internal consistency for the full instrument is not appropriate, due to the questionnaire's multidimensional structure, a previously determined value of $\alpha=0.86$ for the original 21-item ÖMSQ was used to enable comparative analysis.

\section{$\underline{\text { Practical characteristics }}$}

Four practical characteristics (Gabel et al., 2011; Gabel et al., 2012) included: missing responses as calculated from the total number of responses; readability as calculated from the FleschKincaid grade score and reading ease (Kincaid et al., 1975; Paasche-Orlow et al., 2003) and completion and scoring times as calculated respectively from patient and therapist groups. The completion time was averaged from four patients, each with a different regional problem of low back, neck, arm and leg, who completed questionnaires on two occasions providing a total of eight timed completions. Scoring time was the average of two therapists' calculations of each completed questionnaire, a total of eight questionnaires each.

\section{$\underline{\text { Statistical Analysis }}$}

The SPSS version 14.0 (Inc, Chicago, IL) was used with significance level set at $p<0.01$. Factor analysis used MLE with varimax rotation and coefficient suppression at 0.30 (Costello, 2005).

\section{RESULTS}

The psychometric characteristics were compared between both ÖMSQ-12 versions and the original 21-item ÖMSQ (Table 3). Criterion validity was higher for the content-retention (0.97) than the factor analysis version (0.94), but reliability was lower (0.95 versus 0.97$)$. The optimal cut-off score for the predictive ability of a high risk for absenteeism, functional impairment, problem severity and high cost was 60\% (72 ÖMSQ-12 points). The optimal cut-off score for the predictive ability of a low risk for absenteeism and low cost was 48\% (57 ÖMSQ-12 points). The likelihood ratios for convergent validity were comparable or higher for all outcomes except long term absenteeism (Table 3). The two ÖMSQ-12 versions correlated highly $(\mathrm{r}=0.72)$ with the outcome of recovery. 
Table 3: Measurement properties of the ÖMSQ-12 concept-retention and factor analysis versions and the 21-item ÖMSQ in a general musculoskeletal working population

\begin{tabular}{|c|c|c|c|c|c|}
\hline \multicolumn{3}{|c|}{ Measurement Property } & 21-item ÖMSQ & ÖMSQ-12 (CR) & ÖMSQ-12 (FA) \\
\hline \multicolumn{3}{|c|}{ Criterion validity: Pearson's $r(p<0.01)$} & Not applicable & 0.97 & 0.94 \\
\hline \multicolumn{3}{|c|}{ Convergent validity: Likelihood ratio (LR) } & & & \\
\hline \multirow[t]{3}{*}{$\frac{\text { NOT absent }}{(0 \text { days off })}$} & $\frac{\text { ÖMSQ-21 }}{\text { (cut-off) }}$ & $\frac{\text { ÖMSQ-12 }}{\text { (cut-off) }}$ & $\begin{array}{l}\mathrm{LR}= \\
(1-\text { Sensitivity } \\
\end{array}$ & $\begin{array}{l}\text { LK= } \\
\qquad \underline{\text { (1-Specificity })}\end{array}$ & $\begin{array}{l}\text { LK= } \underline{\text { Sensitivity }} \\
\quad \text { (1-Specificity) }\end{array}$ \\
\hline & Points (\%) & Points (\%) & & & \\
\hline & $100(48)$ & $57(48)$ & $80.8: 77.6=3.6$ & $83.3: 76.9=4.6$ & $86.1: 69.2=5.0$ \\
\hline \multicolumn{6}{|l|}{$\underline{\text { Long term }}$} \\
\hline \multirow[t]{2}{*}{ (28 days off) } & $113(54)$ & $66(55)$ & $67.4: 82.1=3.8$ & $73.6: 84.4=3.2$ & $71.7: 93.3=3.3$ \\
\hline & $126(60)$ & $72(60)$ & $71.7: 80.4=3.7$ & $79.2: 71.1=3.4$ & $81.1: 80.0=4.2$ \\
\hline \multicolumn{6}{|l|}{ Function } \\
\hline \multirow[t]{2}{*}{$(\mathrm{PRO})$} & $113(54)$ & $66(55)$ & $89.5: 76.6=3.8$ & $73.1: 82.6=3.1$ & $71.2: 91.3=3.2$ \\
\hline & $126(60)$ & $72(60)$ & $73.7: 79.7=3.6$ & $84.6: 76.1=4.9$ & $80.8: 78.3=4.1$ \\
\hline \multicolumn{5}{|l|}{$\underline{\text { Severity }}$} & $71.2: 91.3=3.2$ \\
\hline$(\mathrm{NRS})$ & $126(60)$ & $72(60)$ & $73.7: 79.7=3.6$ & $84.6: 76.1=4.9$ & $80.8: 78.3=4.1$ \\
\hline \multicolumn{4}{|l|}{$\underline{\text { Cost }}$} & $84.5: 77.8=5.0$ & $85.9: 63.0=4.5$ \\
\hline \multicolumn{5}{|l|}{ Cost } & $61.5: 97.0=2.5$ \\
\hline$(>\$ 10,000)$ & $126(60)$ & $72(60)$ & $70.6: 75.0=2.8$ & $73.8: 78.8=3.0$ & $72.3: 84.8=3.1$ \\
\hline \multicolumn{3}{|c|}{$\begin{array}{l}\text { Divergent validity: } \mathrm{t}-\mathrm{stat} \text { range, } \mathrm{p}<0.001, \text { from } \\
\ddot{O} M S Q \text { scores between known groups outcomes }\end{array}$} & $5.2-7.0$ & $4.8-6.5$ & $5.2-7.4$ \\
\hline \multicolumn{3}{|c|}{ Reliability: ICC $2.1(\mathrm{p}<0.01)$} & 0.97 & 0.95 & 0.97 \\
\hline \multicolumn{3}{|c|}{$\begin{array}{l}\text { Recovery time } \mathbf{t}^{\mathbf{8 0}} \text { : time to reach } 80 \% \text { recovery } \\
\text { (r value correlation with baseline ÖMSQ-12) }\end{array}$} & 0.73 & 0.72 & 0.72 \\
\hline \multicolumn{3}{|c|}{ Factor structure: number extracted with MLE } & 6 & 4 & 4 \\
\hline \multicolumn{3}{|c|}{ Internal consistency: Cronbach's $\alpha$} & 0.86 & 0.75 & 0.75 \\
\hline \multicolumn{3}{|c|}{ Missing responses: (\% missed) } & 5.6 & 4.9 & 4.2 \\
\hline \multicolumn{3}{|c|}{ Readability (Flesch grade, reading ease) } & $6.5,65.5$ & $6.9,63.5$ & $6.9,63.5$ \\
\hline \multicolumn{3}{|c|}{ Completion time: minutes } & $5.57 \pm 3.03$ & $4.42 \pm 2.39$ & $4.42 \pm 2.39$ \\
\hline \multicolumn{3}{|c|}{ Scoring time: (minutes) } & $1.28 \pm 0.10$ & $52 \pm 7$ & $52 \pm 7$ \\
\hline
\end{tabular}

$\mathrm{CR}=$ content retention version; $\mathrm{FA}=$ factor analysis version; $\mathrm{PRO}=$ patient reported outcome

Grey shading enables direct comparison for optimal cut-off scores between the original or shortened versions of the questionnaire, and the related likelihood ratios (LR). LR= Sensitivity/(1-Specificity) 
The factor analysis correlation matrix was determined as suitable from the Kaiser-MeyerOklin value for both ÖMSQ-12 versions and highly significant Barlett Test of Sphericity $(p<0.001)$. They each generated three factors based on the Scree plot (Cattell, 1966), eigenvalues $>1.0$ (Kaiser, 1960) and item-variance $>10 \%$ (Field, 2005). The total cumulative variance was $74 \%$ for the content-retention version and $73 \%$ for the factor analysis version. The rotated threecomponent solution showed different loadings for each version. The qualitative content-retention version had greater consistency with the designated constructs (Table 4) but cross-loading for one item (\#11, recovery expectation). The quantitative factor analysis version (Table 5) showed loading that was less consistent with the designated constructs, particularly fear avoidance and problem.

For internal consistency of the ÖMSQ-12 versions' individual constructs, the contentretention version was mildly preferred over the factor analysis version. A direct comparison between the original 21-item and each of the ÖMSQ-12 versions was not possible as different items were among the five principal constructs. The alpha ranges, of both ÖMSQ-12 versions, were comparable with the content-retention version at 0.72 to 0.73 and the factor-analysis version at 0.71 to 0.73 .

For the practical characteristics, the number of missing responses, the completion and scoring times were comparable between both ÖMSQ-12 versions and lower than the original 21item ÖMSQ. The readability was identical between ÖMSQ-12 versions, but had a marginally higher grade level and slightly more difficult reading ease (Table 3) compared to the original 21item ÖMSQ.

A comparative analysis of the proposed items from the Short Form ÖMPainQ-10 (Linton et al., 2011) indicated lower criterion validity in both samples, (LBP, r=0.93; general musculoskeletal, $r=0.92$ ) The randomly generated ÖMPainQ-10 had a criterion validity of 0.94 in the general musculoskeletal population. The factor analysis of both the ÖMPainQ-10 and the ÖMPainQ-10-random in the general sample showed a five factor model with both instruments' item loading being diverse and not related to the six previously identified constructs. 
A12-item Örebro Screening Questionnaire

Table 4: Factor structure of concept-retention version of the ÖMSQ-12

\begin{tabular}{|c|c|c|c|c|c|}
\hline & & & \multicolumn{3}{|c|}{ Factor } \\
\hline Item & $\begin{array}{c}\text { Question \# } \\
\text { 12-Item } \\
\text { version }\end{array}$ & $\begin{array}{c}\text { Question \# } \\
\text { 21-Item } \\
\text { version }\end{array}$ & $\begin{array}{c}1 \\
\text { Physical, } \\
\text { Fear Avoidance } \\
\text { \& Satisfaction }\end{array}$ & $\begin{array}{c}2 \\
\text { Psyche } \\
\text { \& Other }\end{array}$ & $\begin{array}{c}3 \\
\text { Problem }\end{array}$ \\
\hline ADL \& Social activity & 12 & Q20 & .892 & & \\
\hline Walk or Light Recreation & 11 & Q18 & .727 & & \\
\hline Fear-Avoid: Activity makes worse & 9 & Q14 & .514 & & \\
\hline Fear-Avoid: Stop work/ADL & 10 & Q16 & .489 & & \\
\hline Satisfaction & 8 & Q13 & .349 & & \\
\hline Depression & 6 & Q10 & & .892 & \\
\hline Anxiety & 5 & Q9 & & .738 & \\
\hline Recovery Expectation & 7 & Q11 & & .461 & .305 \\
\hline Burdensomeness & 2 & Q4 & & .376 & \\
\hline Problem Frequency & 4 & Q7 & & & .805 \\
\hline Problem Severity & 3 & Q6 & & & .762 \\
\hline Problem Duration & 1 & Q3 & & & .454 \\
\hline
\end{tabular}

Extraction method: maximum likelihood. Rotation method: varimax with Kaiser Normalization, suppression=0.30

Table 5: Factor structure of factor analysis version of the ÖMSQ-12

\begin{tabular}{|l|c|c|c|c|c|}
\hline \multicolumn{1}{|c|}{ Item } & & & \multicolumn{3}{|c|}{ Factor } \\
\hline & $\begin{array}{c}\text { Question \# } \\
\text { 12-Item } \\
\text { Version } \\
\text { Not Applicable }\end{array}$ & $\begin{array}{c}\text { Question \# } \\
\text { 21-Item } \\
\text { Version }\end{array}$ & $\begin{array}{c}1 \\
\text { Problem } \\
\text { Absenteeism \& } \\
\text { Fear Avoidance }\end{array}$ & $\begin{array}{c}2 \\
\text { Psyche } \\
\text { Other \& } \\
\text { Problem }\end{array}$ & $\begin{array}{c}\text { Physical \& } \\
\text { Fear Avoidance }\end{array}$ \\
\hline Problem Severity & & Q6 & .796 & & \\
Problem Frequency & & Q7 & .741 & & \\
Problem Duration & & Q3 & .533 & & \\
Absenteeism & & Q2 & .425 & & \\
Fear-Avoid: Stop Activity & & Q15 & .393 & & \\
Depression & & Q10 & & .889 & \\
Anxiety & & Q9 & & .774 & \\
Burdensomeness & & Q4 & & .370 & \\
Problem Intensity - acute & & Q5 & & .308 & \\
ADL \& Social & & Q20 & & & .933 \\
Walk Light Rec & & Q18 & & & .700 \\
Fear-Avoid: Stop work/ADL & & Q16 & & & \\
\hline
\end{tabular}

Extraction method: maximum likelihood. Rotation method: varimax with Kaiser Normalization, suppression=0.30. 
A12-item Örebro Screening Questionnaire MAN Ther 2013

\section{DISCUSSION}

\section{Main Findings}

A comparison of the clinimetric properties of the two ÖMSQ-12 questionnaires favored the qualitative concept-retention version over the quantitative factor-analysis version. This was predominantly due to the higher criterion validity demonstrated with the original source 21-item ÖMSQ, and the more appropriately aligned items within the respective constructs under EFA. The quantitative version, however, showed preferred reliability and predictive validity for longterm validity. This finding in favor of the content-retention version was consistent with previous studies that compared qualitative and quantitative reduction methodologies (Beaton et al., 2005; Gummesson et al., 2006; Gabel et al., 2009).

The reliability was slightly reduced in the concept-retention version compared to the factor analysis version which is anticipated based upon the reductive methodology used. The overall internal consistency alpha range of the original 21-item ÖMSQ constructs (0.64 to 0.81$)$ was preferable to both shortened versions. However, this difference is to be anticipated when a questionnaire is shortened. Most importantly, the Alpha values of both ÖMSQ-12 versions were all $>0.70$ and above the $a$-priori $>0.65$ minimum. Compared to the range of the five principal alpha values of the original 21-item ÖMSQ, both ÖMSQ-12 versions were comparable for each construct but had a notably lower whole-instrument internal consistency.

The criterion validity for the ÖMSQ-12 concept-retention version at $r=0.97$ when measured against the original 21-item ÖMSQ was higher than that of the ÖMSQ-12 factor analysis version at 0.94 . Both versions were also higher than the Short Form ÖMPainQ-10 at 0.92, which was itself higher than that reported for the ÖMPainQ-10 in the published literature at 0.91 (Linton et al., 2011). It was most interesting that the ÖMPainQ-10-random version produced a criterion validity at $\mathrm{r}=0.94$, higher than the proposed ÖMPainQ-10 and equivalent to the ÖMSQ-12 factor analysis version. This is important as it confirms that shortened versions of existing questionnaires should not be gauged solely on their criterion validity with the instrument from which they are derived as even a random selection of items can produce high criterion validity (Beaton et al., 2005; Gabel et al., 2009). This reinforces the importance of the methodology used for item selection and that item reduction through the qualitative contentretention process has shown a consistently favorable result over quantitative selection processes (Beaton et al., 2005; Gabel et al., 2009; Gabel et al., 2011). Selected items should represent each 
A12-item Örebro Screening Questionnaire MAN Ther 2013

recognized construct (Coste et al., 1997; Streiner and Norman, 2008). These items, when examined through EFA, should then group under the previously designated constructs. This factor grouping was demonstrated for both ÖMSQ-12 versions developed in this study, but such grouping was not found for the ÖMPainQ-10 versions.

The loading of the factor structure of the ÖMSQ-12 concept-retention version fitted marginally less well than the factor analysis version. This is anticipated as the factor analysis version is derived from the original factor loadings in the 21-item ÖMSQ. The fit of the loading for both ÖMSQ-12 versions was preferable to that shown by either version of the Short Form ÖMPainQ-10 as their items loaded less consistently under the proposed constructs.

Predictive ability cut-off scores were marginally higher for the selected concept-retention ÖMSQ-12 version compared to the original 21-item ÖMSQ. The original 21-item ÖMSQ had a low-risk cut-off of $43 \%$ compared to $48 \%$ for the ÖMSQ-12, while the high risk cut-off ranged from $51 \%$ to $55 \%$ compared to $60 \%$ for the ÖMSQ-12. For all outcomes the likelihood ratios were comparable or higher for the ÖMSQ-12, apart from long term absenteeism. Both ÖMSQ-12 versions had improved practicality, apart from the marginally higher readability, with a lower number of missing responses, shorter completion time and shorter scoring time. The two ÖMSQ12 versions correlation with recovery time at $r=0.72$ was comparable to the $r=0.73$ found for the full 21-item version (Gabel et al., 2012).

\section{Limitations and Strengths}

The limitations include the data being a retrospectively analyzed and that it was specific to workinjured patients over 18 years. Consequently, the study will require prospective validation. Findings cannot be generalized to all patients or to workers beyond six months after their injury.

The strengths include the strong correlation between the ÖMSQ-12 and the original 21item ÖMSQ. This study complies with the recommendations of developing a shortened version of the Örebro instrument with improved practicality and predictive ability compared to the original (Hockings et al., 2008; Melloh et al., 2009; Sattelmayer et al., 2011; Gabel et al., 2011; Gabel et al., 2012). 
A12-item Örebro Screening Questionnaire MAN Ther 2013

\section{$\underline{\text { Implications for Practice }}$}

The improved practicality of this shorter instrument can reduce administrative burden and improve predictive ability, which will consequently increase clinical relevance. This improved practicality will also facilitate early identification of individuals at risk of a delayed recovery due to psychosocial factors. Furthermore, this should also encourage the early provision of targeted intervention strategies that utilize psychosocial informed management principles. The provision of a shortened instrument is supported by the improved prediction cut-off scores that indicated potential delayed recovery. These prediction scores could be used to facilitate intervention choices or assist determination of appropriate care. This may include suitability for rehabilitation through physical or psychological services. In this way the potential for clinicians to adopt this screening instrument should be increased.

\section{$\underline{\text { Implications for Research }}$}

Prospective validation is required in both general working and non-working musculoskeletal populations as well as in sub-region populations, such as LBP, whiplash and regional limb problems. An investigation into the direct relationship between recovery time and baseline screening, that incorporates interactive web-based predictive technology, is also a future goal. It is noted that the 21-item ÖMSQ had correlated at $\mathrm{r}=0.99$ with the original ÖMPainQ (Gabel et al., 2011), suggesting that the ÖMSQ-12 may be directly substituted for the ÖMPainQ and the proposed ÖMPainQ-10 which would open other avenues of research.

\section{CONCLUSION}

The content-retention ÖMSQ-12 is a shortened version of the 21-item ÖMSQ, itself a modification of the original 21-item ÖMPainQ. It has high criterion validity, retention of critical item content, sound psychometric characteristics and improved practicality without the loss of predictive ability. The ÖMSQ-12 may help identify musculoskeletal work-injured patients at risk of prolonged recovery, functional impairment and subsequent high insurer and social costs with lower patient and administrative burden. Prospective validation in a general working and nonworking musculoskeletal population is required. 
Figure 2

\section{Örebro Musculoskeletal Screening Questionnaire 12-Item Short Form (ÖMSQ-12)}

NAME:

Date:

Problem:

1. When did your current pain or problem start? Check $(\checkmark)$ one.
0-1 weeks [1] $\square$ 1-2 weeks
[2]
3-4 weeks [3]
4-5 weeks
[4] $\square$ 6-8 weeks [5]
9-11 weeks [6] $\square$ 3-6 months [7] $\square$ 6-9 months [8] $\square$ 9-12 months [9] $\square$ over 1 year [10]

2. Rate how much of a burden it is to perform all the things you need to do in a normal day. Not at all 2 3 4 5
6
78
910
Extremely

3. For the last 2-3 days, rate on average how bothersome your pain or problem is.

$$
\begin{array}{ccccccccccc}
\begin{array}{c}
\text { 0 } \\
\text { Not at all }
\end{array} & 1 & 2 & 3 & 4 & 5 & 6 & 7 & 8 & 9 & \begin{array}{c}
10 \\
\text { Extremely }
\end{array}
\end{array}
$$

4. For the last 2-3 days, what percentage of the day do you notice your pain or problem?

$\begin{array}{ccccccccccc}\begin{array}{c}0 \\ \text { Never }\end{array} & 10 & 20 & 30 & 40 & 50 & 60 & 70 & 80 & 90 & 100 \\ \text { All the time }\end{array}$

\section{We also need a bit more information on your thoughts and feelings.}

5. During the past 2-3 days, rate how tense or anxious you have felt.

$$
\text { Not at all Extremely }
$$

6. During the past 2-3 days, rate how "depressed" or "down" you have felt.
Not at all
4
5
8
910
Extremely

123

7

7. What do you think is the risk that your current pain or problem will not improve?

$$
\begin{array}{llllllllll}
\text { No risk } & 1 & 2 & 3 & 4 & 5 & 6 & 7 & 8 & 9 \\
\text { Very large risk }
\end{array}
$$

8. Think of your life; rate how satisfied you are with your current situation.

$$
\begin{array}{ccccccccccc}
\mathbf{0} & 1 & 2 & 3 & 4 & 5 & 6 & 7 & 8 & 9 & \begin{array}{c}
10 \\
\text { Not at all }
\end{array} \\
& & & & & & & & & \text { Extremely }
\end{array}
$$

How true are the next two statements for you?

9. Physical activity makes my pain or problem worse.

$$
\begin{array}{ccccccccccc}
\mathbf{0} & 1 & 2 & 3 & 4 & 5 & 6 & 7 & 8 & 9 & \begin{array}{c}
10 \\
\text { Not at all }
\end{array} \\
& & & & & & & & & \text { Extremely }
\end{array}
$$

10. I should not do my normal daily routine or work with my present pain or problem.

$\begin{array}{ccccccccccc}\mathbf{0} & 1 & 2 & 3 & 4 & 5 & 6 & 7 & 8 & 9 & \begin{array}{c}10 \\ \text { Not at all }\end{array} \\ & & & & & & & & & \text { Extremely }\end{array}$

Help us to better understand your current physical abilities. [10-x]

11. I can walk for an hour or participate in my normal light recreational or sporting activities.

$\begin{array}{ccccccccccc}\mathbf{0} & 1 & 2 & 3 & 4 & 5 & 6 & 7 & 8 & \begin{array}{c}9 \\ \text { Completely Normal }\end{array} \\ \text { Not at all } & & & & & & & & & \end{array}$

12. I manage my daily routine and social activities (eg. shopping or transport or see friends).

$\begin{array}{ccccccccccc}\mathbf{0} & 1 & 2 & 3 & 4 & 5 & 6 & 7 & 8 & \begin{array}{c}9 \\ \text { Completely Normal }\end{array} \\ \text { Not at all } & & & & & & & & & \end{array}$

Therapist's Notes: Questions scores $=0$-10, EXCEPT 8, 11\&12 where score $=\mathbf{1 0 - x}$

Scores: $1-7=\ldots ; 9-10=\ldots \quad ; 8,11 \& 12=\ldots \quad$ TOTAL $=$ 
A12-item Örebro Screening Questionnaire MAN Ther 2013

\section{FIGURE LEGENDS}

Figure 1: Flow chart for retrospective development and validation of the ÖMSQ-12

Figure 2: The Short-Form Örebro Musculoskeletal Screening Questionnaire (ÖMSQ-12)

Table 1: Characteristics of the calibration and validation cohorts

Table 2: Items and constructs of concept-retention and factor analysis ÖMSQ-12 versions, ÖMPainQ-10 and ÖMPainQ-10 from random items

Table 3: Measurement properties of the ÖMSQ-12 concept-retention and factor analysis versions and the 21-item ÖMSQ in a general musculoskeletal working population

Table 4: Factor structure of concept-retention version of the ÖMSQ-12

Table 5: Factor structure of factor analysis version of the ÖMSQ-12

\section{REFERENCES}

Beaton DE, Wright JG, Katz JN, Group UEC. Development of the QuickDASH: comparison of three item-reduction approaches. J Bone Joint Surg Am 2005; 87(5): 1038-46.

Boersma K, Linton SJ. How does persistent pain develop? An analysis of the relationship between psychological variables, pain and function across stages of chronicity. Behav Res Ther 2005; 43(11): 1495-507. Epub 2005 Jan 26.

Cattell RB. The scree test for the number of factors. Multivariate Behavioural Research 1966; 1: 24576.

Coste J, Guillemin F, Pouchot J, Fermanian J. Methodological approaches to shortening composite measurement scales. J Clin Epidemiol 1997; 50(3): 247-52.

Costello AB, Osborne J. Best practices in exploratory factor analysis: four recommendations for getting the most from your analysis. Practical Assessment, Research \& Evaluation 2005; 10(7): $1-9$.

Driessen MT, Anema JR, Proper KI, Bongers PM, van der Beek AJ. Stay@Work: Participatory Ergonomics to prevent low back and neck pain among workers: design of a randomised controlled trial to evaluate the (cost-)effectiveness. BMC Musculoskelet Disord 2008; 9: 145.

Field A. Discovering Statistics using SPSS, 2nd ed. London: SAGE Publications Ltd, 2005;

Gabel CP, Burkett B, Neller A, Yelland M. Can long term impairment in General Practitioner Whiplash patients be predicted using screening and Patient Report Outcomes? Int J Rehabil Res 2008; 31(1): 79-80. 
A12-item Örebro Screening Questionnaire MAN Ther 2013

Gabel CP, Burkett B, Yelland M. Balancing Fidelity and Practicality in Short Version Musculoskeletal Outcome Measures Phys Ther Rev 2009; 14(4): 221-5.

Gabel CP, Burkett B, Yelland M, Melloh M, Osborne J. The Örebro Musculoskeletal Screening Questionnaire: validation of a modified primary care musculoskeletal screening tool in an acute work injured population. Man Ther 2012; "In print".

Gabel CP, Melloh M, Yelland M, Burkett B, Roiko A. Predictive Ability of a Modified Örebro Musculoskeletal Pain Questionnaire in an Acute Low Back Pain Working Population. Eur Spine J 2011; 20(3): 449-57.

George D, Mallery P. SPSS for Windows step by step: A simple guide and reference. 11.0 update, 4th ed.. ed. Boston: Allyn \& Bacon., 2003;

Gjesdal S, Bratberg E, Mæland JG. Musculoskeletal Impairments in the Norwegian Working Population: The Prognostic Role of Diagnoses and Socioeconomic Status: A Prospective Study of Sickness Absence and Transition to Disability Pension. Spine 2009; 34(14): 151925.

Gummesson C, Ward MM, Atroshi I. The shortened disabilities of the arm, shoulder and hand questionnaire (QuickDASH): validity and reliability based on responses within the full-length DASH. BMC Musculoskelet Disord 2006; 7: 4.

Hill JC, Dunn KM, Main CJ, Hay EM. Subgrouping low back pain: A comparison of the STarT Back Tool with the Orebro Musculoskeletal Pain Screening Questionnaire. Eur J Pain 2009; 14(1): 83-9.

Hockings RL, McAuley JH, Maher CG. A systematic review of the predictive ability of the Orebro Musculoskeletal Pain Questionnaire. Spine 2008; 33(15): E494-500.

Ijzelenberg W, Burdorf A. Risk factors for musculoskeletal symptoms and ensuing health care use and sick leave. Spine 2005; 30(13): 1550-6.

Kaiser HF. The application of electronic computers to factor analysis. Educ Psychol Meas 1960; 20: 141-51.

Kincaid JP, Fishburne RP, Rogers RL, Chissom BS. Derivation of new readability formulas (Automated Readability Index, Fog Count, and Flesch Reading Ease Formula) for Navy enlisted personnel. Research Branch report., Memphis: Naval Air Station, 1975; pp. 8-75.

Liebenson C, Yeomans S. Assessment of Psychosocial Risk Factors of Chronicity - Yellow Flags. In: Liebenson C editor, Rehabilitation of the Spine: A Practitioner's Manual Baltimore, MD: Lippincott, Wilkins and Williams, 2007; pp. 183-200. 
A12-item Örebro Screening Questionnaire MAN Ther 2013

Linton SJ, Boersma K. Early identification of patients at risk of developing a persistent back problem: the predictive validity of the Orebro Musculoskeletal Pain Questionnaire. Clin J Pain 2003; 19(2): 80-6.

Linton SJ, Nicholas M, MacDonald S. Development of a short form of the Örebro Musculoskeletal Pain Screening Questionnaire. Spine (Phila Pa 1976) 2011; 36(22): 1891-5.

Melloh M, Elfering A, Egli Presland C, Roeder C, Barz T, Rolli Salathé C, Tamcan O, Mueller U, Theis JC. Identification of prognostic factors for chronicity in patients with low back pain: a review of screening instruments. Int Orthop 2009; 33(2): 301-13.

MAA-NSW, Motor Accidents Authority of New South Wales Injury Management Guidelines, Sydney, 2011. Accessed Jan. 2012.

Paasche-Orlow MK, Taylor HA, Brancati FL. Readability Standards for Informed-Consent Forms as Compared with Actual Readability. N Engl J Med 2003; 348(8): 721-6.

Ramond A, Bouton C, Richard I, Roquelaure Y, Baufreton C, Legrand E, Huez JF. Psychosocial risk factors for chronic low back pain in primary care — a systematic review. Fam Pract 2011; 28(1): 12-21.

Sattelmayer S, Lorenz T, Ro“der C, Hilfiker R. Predictive value of the Acute Low Back Pain Screening Questionnaire and the O” rebro Musculoskeletal Pain Screening Questionnaire for persisting problems. Eur Spine J 2011; E-version Pre-publication.

Streiner DL, Norman GR. Health Measurement Scales: A Practical Guide to Their Development and Use, 4 ed. Oxford: Oxford University Press, 2008;

van Tulder M, Becker A, Beckering T, Breen A, Gil del Real MT, Hutchinson A, Koes B, Laerum E, Malmivaara A, On behalf of the COST BB13 Working Group on Guidelines for the Management of Acute Low Back Pain in Primary Care. Chapter 3. European guidelines for the management of acute nonspecific low back pain in primary care. Eur Spine J 2006; 15 (Suppl 2): S169-91.

Westman A, Linton SJ, Ohrvik J, Wahlén P, Leppert J. Do psychosocial factors predict disability and health at a 3-year follow-up for patients with non-acute musculoskeletal pain? A validation of the Orebro Musculoskeletal Pain Screening Questionnaire. Eur J Pain 2008; 12(5): 641-9.

World Health Organisation (WHO). International Classification of Functioning, Disability and Health (ICF), Geneva: World Health Organisation 2001. Accessed Jan. 2012. 